\title{
The Local Stability of Solutions for a Nonlinear Equation
}

\author{
Haibo Yan ${ }^{1,2}$ and Ls Yong ${ }^{1}$ \\ ${ }^{1}$ Department of Applied Mathematics, Southwestern University of Finance and Economics, Chengdu 610074, China \\ ${ }^{2}$ Department of Mathematics, Xinjiang University of Finance and Economics, Urumqi 830012, China
}

Correspondence should be addressed to Haibo Yan; wengxiang00@163.com

Received 15 January 2014; Accepted 19 March 2014; Published 17 April 2014

Academic Editor: Yonghong Wu

Copyright (C) 2014 H. Yan and L. Yong. This is an open access article distributed under the Creative Commons Attribution License, which permits unrestricted use, distribution, and reproduction in any medium, provided the original work is properly cited.

The approach of Kruzkov's device of doubling the variables is applied to establish the local stability of strong solutions for a nonlinear partial differential equation in the space $L^{1}(R)$ by assuming that the initial value only lies in the space $L^{1}(R) \cap L^{\infty}(R)$.

\section{Introduction and Main Results}

Coclite and Karlsen [1] studied the well-posedness for the nonlinear equation

$$
\begin{aligned}
& v_{t}-v_{t x x}+4 h^{\prime}(v) v_{x} \\
& =h^{\prime \prime \prime}(v) v_{x}^{3}+3 h^{\prime \prime}(v) v_{x} v_{x x}+h^{\prime}(v) v_{x x x},
\end{aligned}
$$

where function $h(v)$ satisfies

$$
\left|h^{\prime}(v)\right| \leq c|v|, \quad|h(v)| \leq c|v|^{2},
$$

or

$$
\left|h^{\prime}(v)\right| \leq c, \quad|h(v)| \leq c|v|,
$$

where $c$ is a positive constant. The existence of entropy weak solutions and several other dynamic properties for (1) are investigated in [1].

Consider the following partial differential equation:

$$
\begin{aligned}
& v_{t}-v_{t x x}+m h^{\prime}(v) v_{x} \\
& =h^{\prime \prime \prime}(v) v_{x}^{3}+3 h^{\prime \prime}(v) v_{x} v_{x x}+h^{\prime}(v) v_{x x x},
\end{aligned}
$$

where $m$ is a positive constant and $h(v) \in C^{3}$. If $m=4$ and $h(v)=v^{2} / 2$, (4) becomes the Degasperis-Procesi equation (see [2-12]). If $h(v)=v^{3}$, we note that $h(v)$ does not satisfy conditions (2) and (3). Recently, Wu [13] established the local well-posedness of strong solutions for (4) in the Sobolev space $C\left([0, T) ; H^{s}(R)\right) \cap C^{1}\left([0, T) ; H^{s-1}(R)\right)$ provided that $h(v)=v^{3}$ and the initial value lies in the space $H^{s}(R)$ with $s>3 / 2$. The objective of this work is to study (4) in the case $h(v)=v^{3}$. The local stability of strong solutions for this case is established in the space $L^{1}(R)$. We think that this stability result is a new conclusion for (4).

When $h(v)=v^{3}$, the Cauchy problem of (4) takes the form

$$
\begin{aligned}
v_{t}-v_{t x x}+3 m v^{2} v_{x} & =6 v_{x}^{3}+18 v v_{x} v_{x x}+3 v^{2} v_{x x x}, \\
v(0, x) & =v_{0}(x),
\end{aligned}
$$

which is equivalent to

$$
\begin{gathered}
v_{t}+3 v^{2} v_{x}+(m-1)\left(1-\partial_{x}^{2}\right)^{-1} \partial_{x}\left(v^{3}\right)=0, \\
v(0, x)=v_{0}(x),
\end{gathered}
$$

where the operator $\Lambda^{-2} g=\left(1-\partial_{x}^{2}\right)^{-1} g=(1 / 2) \int_{-\infty}^{\infty} e^{-|x-y|}$ $g(y) d y$ for any $g \in L^{\infty}(R)$ or $g \in L^{p_{0}}(R)$ with a parameter $p_{0}$ satisfying $1 \leq p_{0}<\infty$.

Using the approach of Kruzkov's device of doubling the variables in [14], we obtain the following result.

Theorem 1. Let $v_{1}(t, x)$ and $v_{2}(t, x)$ be two strong solutions of problem (5) or (6) with initial data $v_{1}(0, x)=v_{10}(x) \in L^{1}(R) \cap$ $L^{\infty}(R)$ and $v_{2}(0, x)=v_{20}(x) \in L^{1}(R) \cap L^{\infty}(R)$. Then, for an arbitrary $T>0$,

$$
\begin{array}{r}
\int_{-\infty}^{\infty}\left|v_{1}(t, x)-v_{2}(t, x)\right| d x \leq e^{c t} \int_{-\infty}^{\infty}\left|v_{10}(x)-v_{20}(x)\right| d x, \\
t \in[0, T]
\end{array}
$$

holds, where $c$ is a constant depending on $\left\|v_{10}\right\|_{L^{1}(R)},\left\|v_{10}\right\|_{L^{\infty}(R)}$, $\left\|v_{20}\right\|_{L^{1}(R)},\left\|v_{20}\right\|_{L^{\infty}(R)}$, and $T$. 
This paper is organized as follows. Several lemmas are given in Section 2 and the proof of Theorem 1 is completed in Section 3.

\section{Several Lemmas}

Lemma 1 (see [13]). Assume that initial value $v_{0}=v(0, x) \epsilon$ $L^{2}(R)$. Then the solution of problem (5) with $m>0$ satisfies

$$
\int_{R} K_{1} K d x=\int_{R} \frac{1+\xi^{2}}{m+\xi^{2}}|\widehat{v}(\xi)|^{2} d \xi=\int_{R} \frac{1+\xi^{2}}{m+\xi^{2}}\left|\widehat{v}_{0}(\xi)\right|^{2} d \xi
$$

where $K_{1}=v-\partial_{x x}^{2} v$ and $K=\left(m-\partial_{x x}^{2}\right)^{-1} v$. Moreover, there exist two constants $c_{1}>0$ and $c_{2}>0$ depending only on $m$ such that

$$
c_{1}\left\|v_{0}\right\|_{L^{2}(R)} \leq c_{1}\|v\|_{L^{2}(R)} \leq c_{2}\left\|v_{0}\right\|_{L^{2}(R)} .
$$

In fact, if $v \in L^{1}(R) \cap L^{\infty}(R)$, we know that $v \in L^{2}(R)$.

Lemma 2 (see [13]). Assume that $v_{0} \in L^{2}(R)$. Then

$$
\|v(t, x)\|_{L^{\infty}} \leq\left\|v_{0}\right\|_{L^{\infty}} e^{c t}, \quad \forall t \in[0, \infty)
$$

where $c>0$ is a constant independent of $t$.

Lemma 3. Let $J_{v}=(m-1)\left(1-\partial_{x}^{2}\right)^{-1} \partial_{x}\left(v^{3}\right)$ and $v_{0} \in L^{1}(R) \cap$ $L^{\infty}(R)$. Consider that

$$
\left\|J_{v}\right\|_{L^{\infty}(R)} \leq|m-1|\left\|v_{0}\right\|_{L^{\infty}(R)}^{3} e^{c t}, \quad t \in[0, \infty)
$$

holds, where $c>0$ is a constant independent of $t$.

Proof. Since

$$
\begin{aligned}
\left|\Lambda^{-2} \partial_{x}\left(v^{3}\right)\right| & =\left|\frac{1}{2} \partial_{x} \int_{-\infty}^{\infty} e^{-|x-y|} v^{3}(t, y) d y\right| \\
& =\mid-\frac{1}{2} e^{-x} \int_{-\infty}^{x} e^{y} v^{3}(t, y) d y
\end{aligned}
$$

$$
U_{k}=\frac{1}{k^{2}} \iiint \int_{|(t-\tau) / 2| \leq k, \delta \leq(t+\tau) / 2 \leq T-\delta,|(x-y) / 2| \leq k,|(x+y) / 2| \leq r-\delta}|u(t, x)-u(\tau, y)| d x d t d y d \tau
$$
measurable in cylinder $\Omega=[0, T] \times K_{r}$. If $\delta \in(0, \min [r, T])$ and $h \in(0, \delta)$, then the function

For the Lebesgue point $x_{0}$ of the function $u(x)$, we get $\lim _{k \rightarrow 0} u^{k}\left(x_{0}\right)=u\left(x_{0}\right)$. Since the measure for the set of points which are not the Lebesgue points of $u(x)$ is zero, we have $u^{k}(x) \rightarrow u(x)$ as $k \rightarrow 0$ almost everywhere.

For any $N>0$, let $R_{0}>\sup _{t \in[0, \infty)}\|v\|_{L^{\infty}(R)}^{2}<\infty$. Let $\beth$ represent the cone $\left\{(t, x):|x| \leq N-R_{0} t, 0<t<T_{0}=\right.$ $\left.\min \left(T, N R_{0}^{-1}\right)\right\}$. We let $S_{\tau}$ represent the cross section of the cone $\beth$ by the plane $t=\tau, \tau \in\left[0, T_{0}\right]$. Let $K_{r}=\{x:|x| \leq r\}$, where $r>0$.

Lemma 6. Let $v$ be the strong solution of problem (6) and satisfies $\lim _{k \rightarrow 0} U_{k}=0$.

Lemma 5 (see [14]). If the function $|\partial F(u) / \partial u|$ is bounded, then the function $H(u, v)=\operatorname{sign}(u-v)(F(u)-F(v))$ satisfies the Lipschitz condition in $u$ and $v$, respectively. $\phi(t, x) \in C_{0}^{\infty}\left(\zeta_{T}\right)$. Then

$$
\begin{array}{r}
\iint_{\zeta_{T}}\left\{|v-\alpha| \phi_{t}+\operatorname{sign}(v-k)\left(v^{3}-\alpha^{3}\right) \phi_{x}\right. \\
\left.-\operatorname{sign}(v-\alpha) J_{v}(t, x) \phi\right\} d x d t=0,
\end{array}
$$

where $\alpha$ is an arbitrary constant. 
Proof. Let $\Phi(v)$ be an arbitrary twice smooth function on the line $-\infty<v<\infty$. We multiply the first equation of problem (6) by the function $\Phi^{\prime}(v) \phi(t, x)$, where $\phi(t, x) \in$ $C_{0}^{\infty}\left(\zeta_{T}\right)$. Integrating over $\zeta_{T}$ and transferring the derivatives with respect to $t$ and $x$ to the test function $\phi$, for any constant $\alpha$, we obtain

$$
\begin{array}{r}
\iint_{\zeta_{T}}\left\{\Phi(v) \phi_{t}+\left[\int_{\alpha}^{v} \Phi^{\prime}(z) 3 z^{2} d z\right] \phi_{x}\right. \\
\left.-\Phi^{\prime}(v) J_{v}(t, x) \phi\right\} d x d t=0,
\end{array}
$$

in which we have used $\int_{-\infty}^{\infty}\left[\int_{\alpha}^{v} \Phi^{\prime}(z) 3 z^{2} d z\right] \phi_{x} d x=$ $-\int_{-\infty}^{\infty}\left[\phi \Phi^{\prime}(v) 3 v^{2} v_{x}\right] d x$.

Integration by parts yields

$$
\begin{aligned}
\int_{-\infty}^{\infty}\left[\int_{\alpha}^{v} \Phi^{\prime}(z) 3 z^{2} d z\right] \phi_{x} d x \\
=\int_{-\infty}^{\infty}\left[\left(v^{3}-\alpha^{3}\right) \Phi^{\prime}(v)\right. \\
\left.-\int_{\alpha}^{v}\left(z^{3}-\alpha^{3}\right) \Phi^{\prime \prime}(z) d z\right] \phi_{x} d x
\end{aligned}
$$

Let $\Phi^{k}(v)$ be an approximation of the function $|v-\alpha|$ and set $\Phi(v)=\Phi^{k}(v)$. Using the properties of the $\operatorname{sign}(v-\alpha),(18)$, and (19) and sending $k \rightarrow 0$, we have

$$
\begin{gathered}
\iint_{\zeta_{T}}\left\{|v-\alpha| \phi_{t}+\operatorname{sign}(v-\alpha)\left[v^{3}-\alpha^{3}\right] \phi_{x}\right. \\
\left.-\operatorname{sign}(v-\alpha) J_{v}(t, x) \phi\right\} d x d t=0,
\end{gathered}
$$

which completes the proof.

We note that the proof of (17) can also be found in [14].

Lemma 7. Assume that $v_{1}(t, x)$ and $v_{2}(t, x)$ are two strong solutions of problem (6) associated with the initial data $v_{10}=$ $v_{1}(0, x) \in L^{1}(R) \cap L^{\infty}(R)$ and $v_{20}=v_{2}(0, x) \in L^{1}(R) \cap L^{\infty}(R)$. For any $\phi \in C_{0}^{\infty}\left(\zeta_{T}\right)$,

$$
\begin{aligned}
& \left|\int_{-\infty}^{\infty} \operatorname{sign}\left(v_{1}-v_{2}\right)\left[J_{v_{1}}(t, x)-J_{v_{2}}(t, x)\right] \phi d x\right| \\
& \quad \leq c \int_{-\infty}^{\infty}\left|v_{1}-v_{2}\right| d x
\end{aligned}
$$

holds, where $c$ depends on $\left\|v_{10}\right\|_{L^{1}(R)},\left\|v_{10}\right\|_{L^{\infty}(R)},\left\|v_{20}\right\|_{L^{1}(R)}$, $\left\|v_{20}\right\|_{L^{\infty}(R)}, T$, and $\phi$.

Proof. We obtain

$$
\begin{aligned}
\Lambda^{-2} & {\left[v_{1}^{3}(t, x)-v_{2}^{3}(t, x)\right] } \\
& =\frac{1}{2} \int_{-\infty}^{\infty} e^{-|x-y|}\left[v_{1}^{3}(t, y)-v_{2}^{3}(t, y)\right] d y,
\end{aligned}
$$

$$
\begin{aligned}
& \mid \Lambda^{-2} \partial_{x} {\left[v_{1}^{3}(t, x)-v_{2}^{3}(t, x)\right] \mid } \\
&=\mid-\frac{1}{2} e^{-x} \int_{-\infty}^{x} e^{y}\left[v_{1}^{3}(t, y)-v_{2}^{3}(t, y)\right] d y \\
& \quad+\frac{1}{2} e^{x} \int_{x}^{\infty} e^{-y}\left[v_{1}^{3}(t, y)-v_{2}^{3}(t, y)\right] d y \mid \\
& \leq c \int_{-\infty}^{\infty} e^{-|x-y|}\left|v_{1}^{3}(t, y)-v_{2}^{3}(t, y)\right| d y \\
& \leq c \int_{-\infty}^{\infty} e^{-|x-y|}\left|v_{1}(t, y)-v_{2}(t, y)\right| d y,
\end{aligned}
$$

in which we have used Lemma 2. Using (23) and the Fubini theorem completes the proof.

\section{Proof of Theorem 1}

Here we state that the techniques used in this paper to establish the local stability of solutions for problem (6) come from the methods of Kruzkov's device of doubling the variables presented in Kruzkov's paper [14].

Proof of Theorem 1. For an arbitrary $T>0$, set $\zeta_{T}=[0, T] \times R$. Let $f(t, x) \in C_{0}^{\infty}\left(\zeta_{T}\right)$. We assume that $f(t, x)=0$ outside the cylinder

$$
\Theta=\{(t, x)\}=[\delta, T-2 \delta] \times H_{r-2 \delta}, \quad 0<2 \delta \leq \min (T, r) .
$$

We define

$$
\begin{aligned}
h & =f\left(\frac{t+\tau}{2}, \frac{x+y}{2}\right) \theta_{k}\left(\frac{t-\tau}{2}\right) \theta_{k}\left(\frac{x-y}{2}\right) \\
& =f(\cdots) \lambda_{k}(*),
\end{aligned}
$$

where $(\cdots)=((t+\tau) / 2,(x+y) / 2)$ and $(*)=((t-\tau) / 2,(x-$ $y) / 2$ ). The function $\theta_{k}(\omega)$ is defined in (13). Note that

$$
h_{t}+h_{\tau}=f_{t}(\cdots) \lambda_{k}(*), \quad h_{x}+h_{y}=f_{x}(\cdots) \lambda_{k}(*) .
$$

Taking $v=v_{1}(t, x)$ and $\alpha=v_{2}(\tau, y)$ and assuming that $f(t, x)=0$ outside the cylinder $\Theta$, from Lemma 6 , we have

$$
\begin{aligned}
\iiint \int_{\zeta_{T} \times \zeta_{T}}\{ & \left|v_{1}(t, x)-v_{2}(\tau, y)\right| h_{t} \\
& +\operatorname{sign}\left(v_{1}(t, x)-v_{2}(\tau, y)\right) \\
& \times\left(v_{1}^{3}(t, x)-v_{2}^{3}(\tau, y)\right) h_{x} \\
& -\operatorname{sign}\left(v_{1}(t, x)-v_{2}(\tau, y)\right) \\
& \left.\times J_{v_{1}}(t, x) h\right\} d x d t d y d \tau=0 .
\end{aligned}
$$


Similarly, it has

$$
\begin{aligned}
\iiint \int_{\zeta_{T} \times \zeta_{T}}\{ & \left|v_{2}(\tau, y)-v_{1}(t, x)\right| g_{\tau} \\
& +\operatorname{sign}\left(v_{2}(\tau, y)-v_{1}(t, x)\right) \\
& \times\left(v_{2}^{3}(\tau, y)-v_{1}^{3}(t, x)\right) h_{y} \\
& -\operatorname{sign}\left(v_{2}(\tau, y)-v_{1}(t, x)\right) \\
& \left.\times J_{v_{2}}(\tau, y) h\right\} d x d t d y d \tau=0,
\end{aligned}
$$

from which we obtain

$$
\begin{aligned}
& 0 \leq \iiint \int_{\zeta_{T} \times \zeta_{T}}\left|v_{1}(t, x)-v_{2}(\tau, y)\right|\left(h_{t}+h_{\tau}\right) d x d t d y d \tau \\
& +\iiint \int_{\zeta_{T} \times \zeta_{T}} \operatorname{sign}\left(v_{1}(t, x)-v_{2}(\tau, y)\right) \\
& \quad \times\left(v_{1}^{3}(t, x)-v_{2}^{3}(\tau, y)\right) \\
& \quad \times\left[h_{x}+h_{y}\right] d x d t d y d \tau \\
& +\mid \iiint \int_{\zeta_{T} \times \zeta_{T}} \operatorname{sign}\left(v_{1}(t, x)-v_{2}(\tau, y)\right) \\
& \quad \times\left(J_{v_{1}}(t, x)-J_{v_{2}}(\tau, y)\right) h d x d t d y d \tau \mid \\
& \quad \iiint \int_{\zeta_{T} \times \zeta_{T}}\left(I_{1}+I_{2}\right) d x d t d y d \tau \\
& \quad+\left|\iiint \int_{\zeta_{T} \times \zeta_{T}} I_{3} d x d t d y d \tau\right| .
\end{aligned}
$$

We claim that

$$
\begin{gathered}
0 \leq \iint_{\zeta_{T}}\left|v_{1}(t, x)-v_{2}(t, x)\right| f_{t}+\operatorname{sign}\left(v_{1}(t, x)-v_{2}(t, x)\right) \\
\quad \times\left(v_{1}^{3}(t, x)-v_{2}^{3}(t, x)\right) f_{x} d x d t
\end{gathered}
$$

$$
\begin{aligned}
+\mid \iint_{\zeta_{T}} & \operatorname{sign}\left(v_{1}(t, x)-v_{2}(t, x)\right) \\
& \quad \times\left[J_{v_{1}}(t, x)-J_{v_{2}}(t, x)\right] f d x d t \mid .
\end{aligned}
$$

We note that the first two terms in the integrand of (29) can be represented in the form

$$
H_{k}=H\left(t, x, \tau, y, v_{1}(t, x), v_{2}(\tau, y)\right) \lambda_{k}(*) .
$$

From Lemma 5, we know that $H_{k}$ satisfies the Lipschitz condition in $v_{1}$ and $v_{2}$, respectively. By the choice of $h$, we have $H_{k}=0$ outside the region as follows:

$$
\begin{aligned}
\{(t, x ; \tau, y)\}= & \left\{\delta \leq \frac{t+\tau}{2} \leq T-2 \delta, \frac{|t-\tau|}{2} \leq k,\right. \\
& \left.\frac{|x+y|}{2} \leq r-2 \delta, \frac{|x-y|}{2} \leq k\right\},
\end{aligned}
$$

$\iiint \int_{\zeta_{T} \times \zeta_{T}} H_{k} d x d t d y d \tau$

$$
\begin{aligned}
&=\iiint \int_{\zeta_{T} \times \zeta_{T}}[ H\left(t, x, \tau, y, v_{1}(t, x), v_{2}(\tau, y)\right) \\
&\left.-H\left(t, x, t, x, v_{1}(t, x), v_{2}(t, x)\right)\right] \\
& \times \lambda_{k}(*) d x d t d y d \tau \\
&+\iiint \int_{\zeta_{T} \times \zeta_{T}} H\left(t, x, t, x, v_{1}(t, x), v_{2}(t, x)\right)
\end{aligned}
$$$$
\times \lambda_{k}(*) d x d t d y d \tau
$$

$$
=B_{1}(k)+B_{2} \text {. }
$$

Considering the estimate $|\lambda(*)| \leq c / k^{2}$ and the expression of function $H_{k}$, we have

$$
\left|B_{1}(k)\right| \leq c\left[k+\frac{1}{k^{2}} \iiint \int_{|(t-\tau) / 2| \leq k, \delta \leq(t+\tau) / 2 \leq T-\delta,|(x-y) / 2| \leq k,|(x+y) / 2| \leq r-\delta}\left|v_{2}(t, x)-v_{2}(\tau, y)\right| d x d t d y d \tau\right]
$$

where the constant $c$ does not depend on $k$. Using Lemma 4, we obtain $B_{1}(k) \rightarrow 0$ as $k \rightarrow 0$. The integral $B_{2}$ does not depend on $k$. In fact, substituting $t=\alpha_{1},(t-\tau) / 2=\beta, x=\eta$, $(x-y) / 2=\xi$ and noting that

$$
\int_{-k}^{k} \int_{-\infty}^{\infty} \lambda_{k}(\beta, \xi) d \xi d \beta=1
$$

we have

$$
\begin{aligned}
B_{2}=2^{2} \iint_{\zeta_{T}} H_{k}\left(\alpha_{1}, \eta, \alpha_{1}, \eta, v_{1}\left(\alpha_{1}, \eta\right), v_{2}\left(\alpha_{1}, \eta\right)\right) \\
\quad \times\left\{\int_{-k}^{k} \int_{-\infty}^{\infty} \lambda_{k}(\beta, \xi) d \xi d \beta\right\} d \eta d \alpha_{1} \\
=4 \iint_{\zeta_{T}} H\left(t, x, t, x, v_{1}(t, x), v_{2}(t, x)\right) d x d t .
\end{aligned}
$$


Hence

$$
\begin{aligned}
& \lim _{k \rightarrow 0} \iiint \int_{\zeta_{T} \times \zeta_{T}} H_{k} d x d t d y d \tau \\
& \quad=4 \iint_{\zeta_{T}} H\left(t, x, t, x, v_{1}(t, x), v_{2}(t, x)\right) d x d t
\end{aligned}
$$

Since

$$
\begin{aligned}
I_{3}= & \operatorname{sign}\left(v_{1}(t, x)-v_{2}(\tau, y)\right) \\
& \times\left(J_{v_{1}}(t, x)-J_{v_{2}}(\tau, y)\right) f \lambda_{k}(*),
\end{aligned}
$$

$$
\left|C_{1}(k)\right| \leq c\left(k+\frac{1}{k^{2}} \iiint \int_{|(t-\tau) / 2| \leq k, \delta \leq(t+\tau) / 2 \leq T-\delta,|(x-y) / 2| \leq k,|(x+y) / 2| \leq r-\delta}\left|J_{v_{2}}(t, x)-J_{v_{2}}(\tau, y)\right| d x d t d y d \tau\right)
$$

By Lemmas 3 and 4 , we have $C_{1}(h) \rightarrow 0$ as $k \rightarrow 0$. Using (34), we have

$$
\begin{aligned}
C_{2}= & 2^{2} \iint_{\zeta_{T}} I_{3}\left(\alpha_{1}, \eta, \alpha_{1}, \eta, v_{1}\left(\alpha_{1}, \eta\right), v_{2}\left(\alpha_{1}, \eta\right)\right) \\
& \times\left\{\int_{-h}^{h} \int_{-\infty}^{\infty} \lambda_{h}(\beta, \xi) d \xi d \beta\right\} d \eta d \alpha_{1} \\
= & 4 \iint_{\zeta_{T}} I_{3}\left(t, x, t, x, v_{1}(t, x), v_{2}(t, x)\right) d x d t \\
= & 4 \iint_{\zeta_{T}} \operatorname{sign}\left(v_{1}(t, x)-v_{2}(t, x)\right) \\
& \times\left(J_{v_{1}}(t, x)-J_{v_{2}}(t, x)\right) f(t, x) d x d t .
\end{aligned}
$$

From (36) and (38)-(40), we prove that inequality (30) holds.

Let

$$
B(t)=\int_{-\infty}^{\infty}\left|v_{1}(t, x)-v_{2}(t, x)\right| d x
$$

We define

$$
\rho_{k}(\sigma)=\int_{-\infty}^{\sigma} \theta_{k}(\omega) d \omega, \quad\left(\rho_{k}^{\prime}(\sigma)=\theta_{k}(\sigma) \geq 0\right)
$$

and choose the two numbers $\tau_{1}$ and $\tau_{2} \in\left(0, T_{0}\right), \tau_{1}<\tau_{2}$. In (30), we choose

$$
\begin{array}{r}
f=\left[\rho_{k}\left(t-\tau_{1}\right)-\rho_{k}\left(t-\tau_{2}\right)\right] \chi(t, x), \\
k<\min \left(\tau_{1}, T_{0}-\tau_{2}\right),
\end{array}
$$

where

$$
\begin{array}{r}
\chi(t, x)=\chi_{\varepsilon}(t, x)=1-\rho_{\varepsilon}\left(|x|+R_{0} t-N+\varepsilon\right), \\
\varepsilon>0 .
\end{array}
$$

When $\varepsilon$ is sufficiently small, we note that function $\chi(t, x)=0$ outside the cone $\beth$ and $f(t, x)=0$ outside the set $\Theta$. For $(t, x) \in \Theta$, we have

$$
0=\chi_{t}+R_{0}\left|\chi_{x}\right| \geq \chi_{t}+R_{0} \chi_{x}
$$

Applying (30) and (42)-(45) and suitably choosing large $R_{0}$, we have the inequality

$$
\begin{aligned}
0 \leq \iint_{\xi_{T_{0}}}\left\{\left[\theta_{k}\left(t-\tau_{1}\right)-\theta_{k}\left(t-\tau_{2}\right)\right]\right. \\
\left.\quad \times \chi_{\varepsilon}\left|v_{1}(t, x)-v_{2}(t, x)\right|\right\} d x d t \\
+\mid \iint_{\xi_{T_{0}}}\left[\rho_{k}\left(t-\tau_{1}\right)-\rho_{k}\left(t-\tau_{2}\right)\right]\left[J_{v_{1}}(t, x)-J_{v_{2}}(t, x)\right] \\
\quad \times E(t, x) \chi(t, x) d x d t \mid,
\end{aligned}
$$

where $E(t, x)=\operatorname{sign}\left[v_{1}(t, x)-v_{2}(t, x)\right]$.

From (46), we obtain

$$
\begin{aligned}
0 \leq & \iint_{\xi_{T_{0}}}\left\{\left[\theta_{k}\left(t-\tau_{1}\right)-\theta_{k}\left(t-\tau_{2}\right)\right]\right. \\
& \left.\times \chi_{\varepsilon}\left|v_{1}(t, x)-v_{2}(t, x)\right|\right\} d x d t \\
& +\int_{0}^{T_{0}}\left(\rho_{k}\left(t-\tau_{1}\right)-\rho_{k}\left(t-\tau_{2}\right)\right) \\
& \times\left|\int_{-\infty}^{\infty}\left[J_{v_{1}}(t, x)-J_{v_{2}}(t, x)\right] E(t, x) \chi(t, x) d x\right| d t .
\end{aligned}
$$

Using Lemma 7, we have

$$
\begin{aligned}
& 0 \leq \iint_{\xi_{T_{0}}}\left\{\left[\theta_{k}\left(t-\tau_{1}\right)-\theta_{k}\left(t-\tau_{2}\right)\right]\right. \\
& \left.\quad \times \chi_{\varepsilon}\left|v_{1}(t, x)-v_{2}(t, x)\right|\right\} d x d t \\
& \quad+c \int_{0}^{T_{0}}\left(\rho_{k}\left(t-\tau_{1}\right)-\rho_{k}\left(t-\tau_{2}\right)\right) \\
& \quad \times \int_{-\infty}^{\infty}\left|v_{1}-v_{2}\right| d x d t,
\end{aligned}
$$


Letting $\varepsilon \rightarrow 0$ in (48) and sending $N \rightarrow \infty$, we have

$$
\begin{aligned}
0 \leq \iint_{\xi_{T_{0}}}\left\{\left[\theta_{k}\left(t-\tau_{1}\right)-\theta_{k}\left(t-\tau_{2}\right)\right]\right. \\
\left.\quad \times\left|v_{1}(t, x)-v_{2}(t, x)\right|\right\} d x d t \\
\quad+c \int_{0}^{T_{0}}\left(\rho_{k}\left(t-\tau_{1}\right)-\rho_{k}\left(t-\tau_{2}\right)\right) \\
\quad \times \int_{-\infty}^{\infty}\left|v_{1}-v_{2}\right| d x d t .
\end{aligned}
$$

By the properties of the function $\theta_{k}(\omega)$ for $k \leq \min \left(\tau_{1}, T_{0}-\right.$ $\left.\tau_{1}\right)$, we have

$$
\begin{aligned}
& \left|\int_{0}^{T_{0}} \theta_{k}\left(t-\tau_{1}\right) B(t) d t-B\left(\tau_{1}\right)\right| \\
& \quad=\left|\int_{0}^{T_{0}} \theta_{k}\left(t-\tau_{1}\right)\left[B(t)-B\left(\tau_{1}\right)\right] d t\right| \\
& \quad \leq c \frac{1}{k} \int_{\tau_{1}-k}^{\tau_{1}+k}\left|B(t)-B\left(\tau_{1}\right)\right| d t \longrightarrow 0 \text { as } k \longrightarrow 0,
\end{aligned}
$$

where $c$ is independent of $h$.

Set

$$
P\left(\tau_{1}\right)=\int_{0}^{T_{0}} \rho_{k}\left(t-\tau_{1}\right) B(t) d t=\int_{0}^{T_{0}} \int_{-\infty}^{t-\tau_{1}} \theta_{k}(\sigma) d \sigma B(t) d t
$$

Using the similar proof of (50), we get

$$
P^{\prime}\left(\tau_{1}\right)=-\int_{0}^{T_{0}} \theta_{k}\left(t-\tau_{1}\right) B(t) d t \longrightarrow-B\left(\tau_{1}\right)
$$

$$
\text { as } k \longrightarrow 0 \text {, }
$$

from which we obtain

$$
P\left(\tau_{1}\right) \longrightarrow P(0)-\int_{0}^{\tau_{1}} B(\sigma) d \sigma \quad \text { as } k \longrightarrow 0
$$

Similarly, we have

$$
P\left(\tau_{2}\right) \longrightarrow P(0)-\int_{0}^{\tau_{2}} B(\sigma) d \sigma \quad \text { as } k \longrightarrow 0 .
$$

Then, we get

$$
P\left(\tau_{1}\right)-P\left(\tau_{2}\right) \longrightarrow \int_{\tau_{1}}^{\tau_{2}} B(\sigma) d \sigma \quad \text { as } k \longrightarrow 0 .
$$

Letting $\tau_{1} \rightarrow 0$ and $\tau_{2} \rightarrow t$, from (49), (50), and (55), for any $t \in\left[0, T_{0}\right]$, we have

$$
\begin{aligned}
\int_{-\infty}^{\infty}\left|v_{1}(t, x)-v_{2}(t, x)\right| d x \leq & \int_{-\infty}^{\infty}\left|v_{1}(0, x)-v_{2}(0, x)\right| d x \\
& +c \int_{0}^{t} \int_{-\infty}^{\infty}\left|v_{1}-v_{2}\right| d x
\end{aligned}
$$

where $c$ depends on $\left\|v_{10}\right\|_{L^{1}(R)},\left\|v_{10}\right\|_{L^{\infty}(R)},\left\|v_{20}\right\|_{L^{1}(R)}$, $\left\|v_{20}\right\|_{L^{\infty}(R)}$, and $T$. Using the Gronwall inequality and (56) completes the proof of Theorem 1 .

\section{Conflict of Interests}

The authors declare that there is no conflict of interests regarding the publication of this paper.

\section{Acknowledgments}

This work is supported by both the Fundamental Research Funds for the Central Universities (JBK120504) and the Applied and Basic Project of Sichuan Province (2012JY0020).

\section{References}

[1] G. M. Coclite and K. H. Karlsen, "On the well-posedness of the Degasperis-Procesi equation," Journal of Functional Analysis, vol. 233, no. 1, pp. 60-91, 2006.

[2] A. Constantin and D. Lannes, "The hydrodynamical relevance of the Camassa-Holm and Degasperis-Procesi equations," Archive for Rational Mechanics and Analysis, vol. 192, no. 1, pp. 165-186, 2009.

[3] J. Escher, Y. Liu, and Z. Y. Yin, "Global weak solutions and blowup structure for the Degasperis-Procesi equation," Journal of Functional Analysis, vol. 241, no. 2, pp. 457-485, 2006.

[4] Y. Fu, Y. Liu, and C. Z. Qu, "On the blow-up structure for the generalized periodic Camassa-Holm and Degasperis-Procesi equations," Journal of Functional Analysis, vol. 262, no. 7, pp. 3125-3158, 2012.

[5] D. Henry, "Infinite propagation speed for the DegasperisProcesi equation," Journal of Mathematical Analysis and Applications, vol. 311, no. 2, pp. 755-759, 2005.

[6] G. L. Gui, Y. Liu, and L. X. Tian, "Global existence and blowup phenomena for the peakon $b$-family of equations," Indiana University Mathematics Journal, vol. 57, no. 3, pp. 1209-1234, 2008.

[7] Y. Liu and Z. Y. Yin, "Global existence and blow-up phenomena for the Degasperis-Procesi equation," Communications in Mathematical Physics, vol. 267, no. 3, pp. 801-820, 2006.

[8] S. Y. Lai and Y. H. Wu, "A model containing both the CamassaHolm and Degasperis-Procesi equations," Journal of Mathematical Analysis and Applications, vol. 374, no. 2, pp. 458-469, 2011.

[9] S. Lai and A. Y. Wang, "The well-posedness of solutions for a generalized shallow water wave equation," Abstract and Applied Analysis, vol. 2012, Article ID 872187, 15 pages, 2012.

[10] S. Y. Lai, "The global weak solution for a generalized CamassaHolm equation," Abstract and Applied Analysis, vol. 2013, Article ID 838302, 6 pages, 2013.

[11] Z. W. Lin and Y. Liu, "Stability of peakons for the DegasperisProcesi equation," Communications on Pure and Applied Mathematics, vol. 62, no. 1, pp. 125-146, 2009.

[12] O. G. Mustafa, "A note on the Degasperis-Procesi equation," Journal of Nonlinear Mathematical Physics, vol. 12, no. 1, pp. 1014, 2005.

[13] M. Wu, "The local strong solutions and global weak solutions for a nonlinear equation," Abstract and Applied Analysis, vol. 2013, Article ID 619068, 5 pages, 2013.

[14] S. N. Kruzkov, "First order quasi-linear equations in several independent variables," Mathematics of the USSR-Sbornik, vol. 10, pp. 217-243, 1970. 


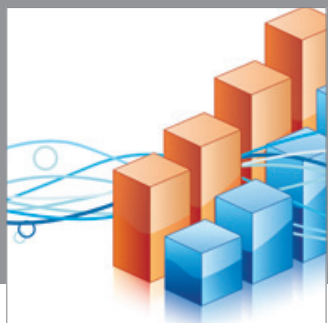

Advances in

Operations Research

mansans

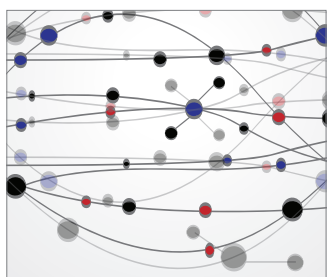

The Scientific World Journal
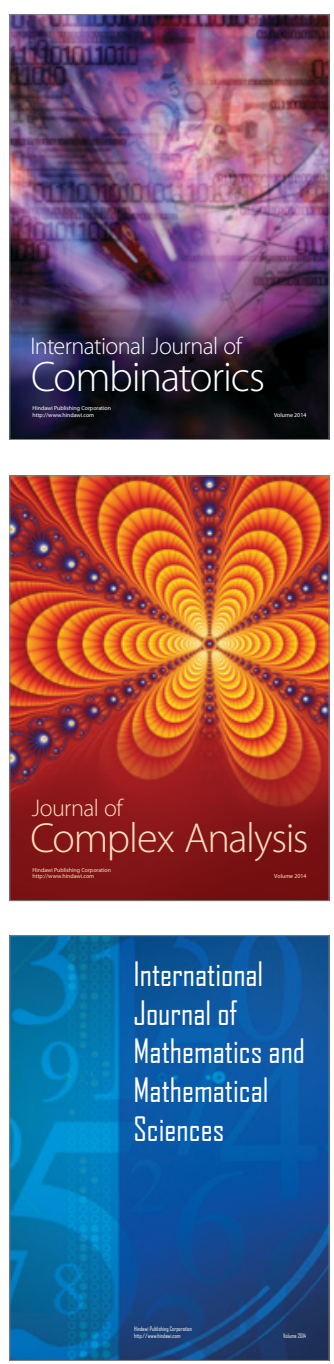
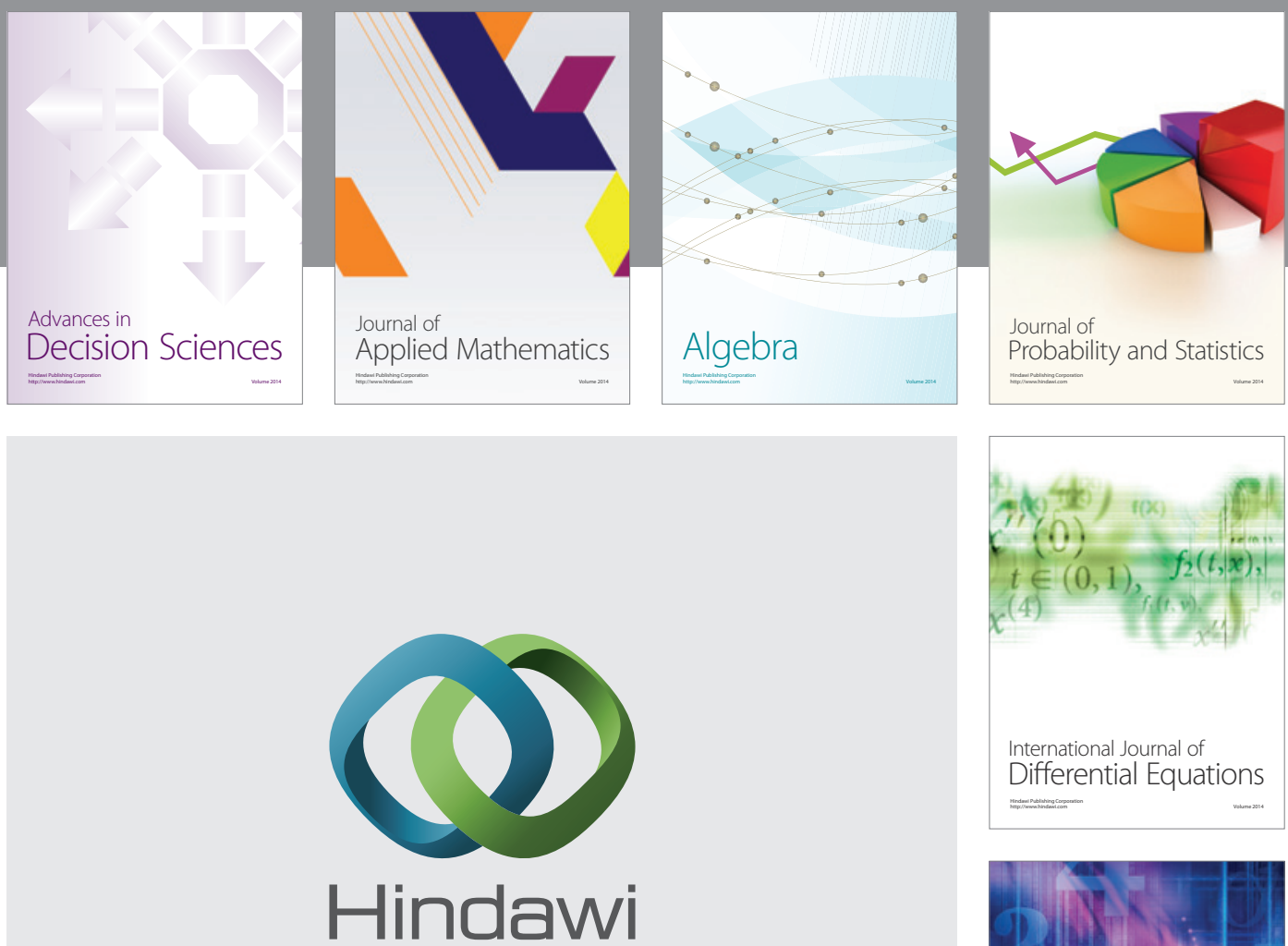

Submit your manuscripts at http://www.hindawi.com
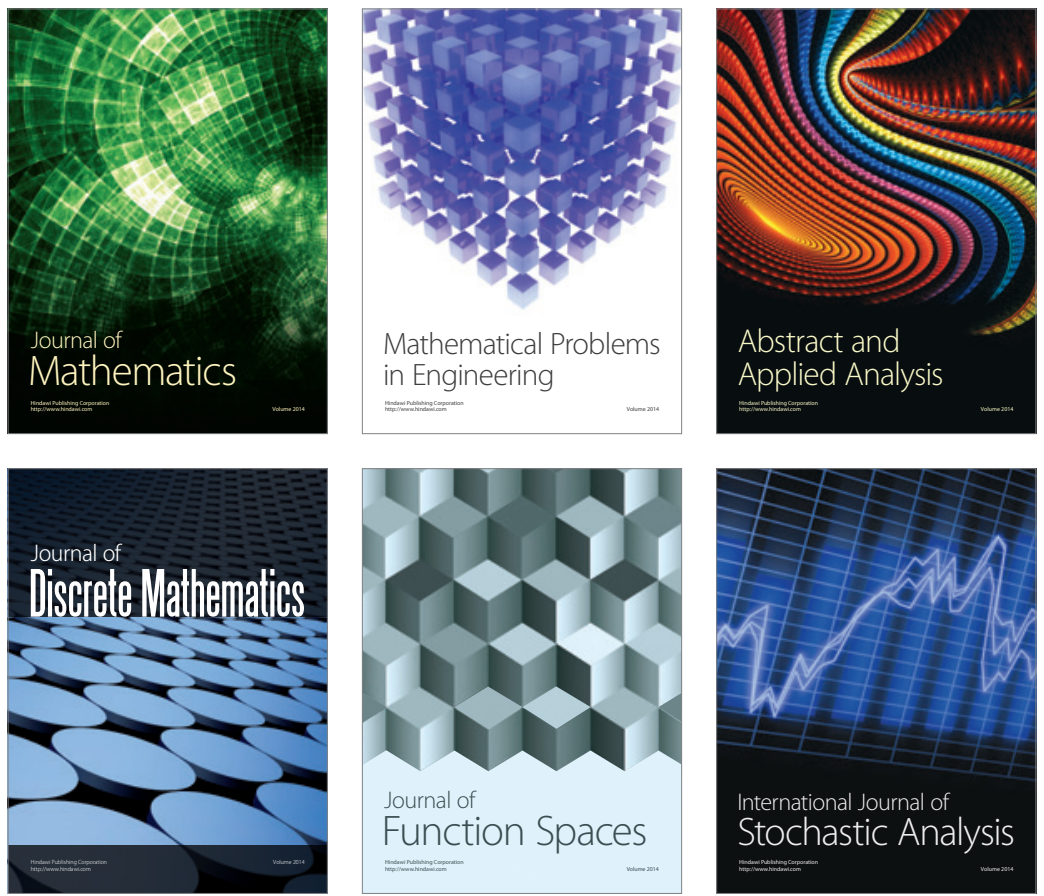

Journal of

Function Spaces

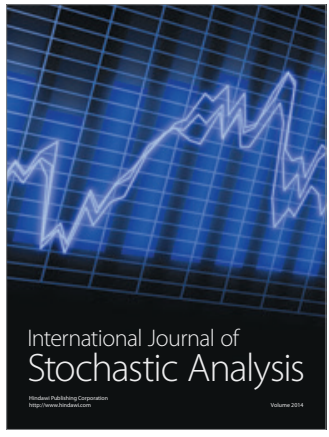

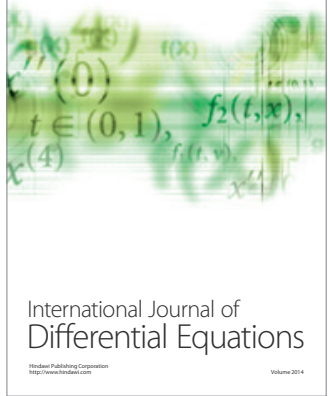
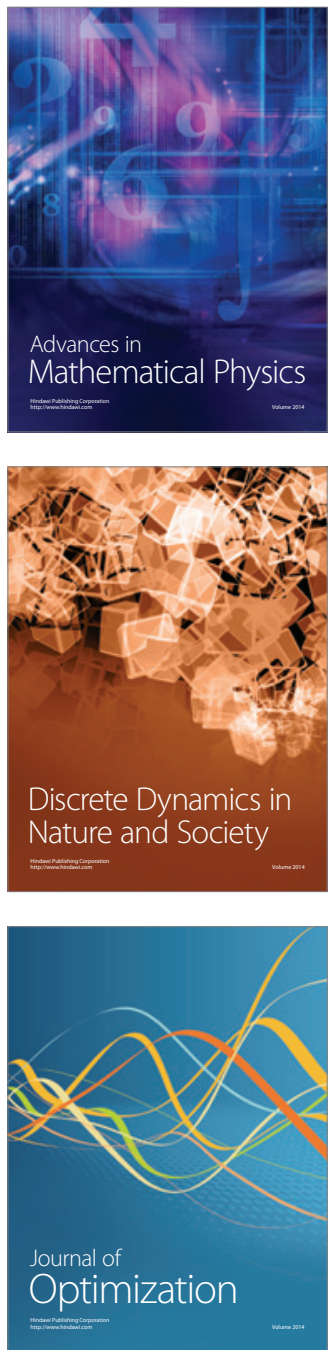